\title{
Der p 2 Recombinant Bacille Calmette-Guérin Targets Dendritic Cells to Inhibit Allergic Airway Inflammation in a Mouse Model of Asthma
}

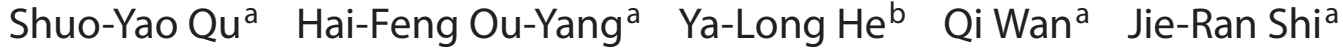 \\ Chang-Gui Wu ${ }^{\mathrm{a}}$ \\ Departments of ${ }^{\mathrm{a}}$ Respiratory Medicine and ${ }^{\mathrm{b}}$ Neurosurgery, Xijing Hospital, Fourth Military Medical University, \\ Xi'an, China
}

\section{Key Words}

Airway inflammation $\cdot$ Dendritic cells $\cdot$ Recombinant bacille Calmette-Guérin $\cdot$ Regulatory $T$ cells

\begin{abstract}
Background: Previous studies showed that a recombinant bacille Calmette-Guérin ( $\mathrm{rBCG}$ ) which expressed the Der $\mathrm{p} 2$ of house dust mites (Der $\mathrm{p} 2 \mathrm{rBCG}$ ) could suppress asthmatic airway inflammation. There are two possible mechanisms: (1) Der $p 2$ rBCG elicits immune deviation from Th2 to Th1, and (2) Der p 2 rBCG induces antigen-specific regulatory $T$ cells. However, the role of dendritic cell (DC) Der p 2 rBCG in this protective effect and in reprogramming T-cell commitment still needs to be studied. Objectives: The aim of this study was to determine whether DCs play a central role in the Der p 2 rBCG-mediated inhibition of allergic airway inflammation. Methods: DCs were collected from Der $\mathrm{p} 2$ rBCG-immunized mice (Der $\mathrm{p} 2 \mathrm{rBCG}-\mathrm{DCs}$ ) and adoptively transferred to Der $p$ 2-sensitized mice. The effects of DCs on airway inflammation and immune regulation were analyzed. Results: Adoptive transfer of DCs from Der $\mathrm{p} 2 \mathrm{rBCG}$-immunized mice suppressed asthmatic responses, including airway inflammation, mucin secretion and airway responsiveness. Der $\mathrm{p} 2$ rBCG-DCs could effectively inhibit excessive Th2 immune responses and induced a subtype of CD4+CD25+Foxp3+ anti-
\end{abstract}

specific regulatory $T$ cells in this asthma model. Furthermore, Der $\mathrm{p} 2$ rBCG immunization recruited more plasmacytoid DCs in abdominal draining lymph nodes. Conclusions: These findings suggest that DCs played a key role in Der $\mathrm{p} 2$ rBCG-induced immunoregulation. Compared with BCG, Der p 2 rBCG displayed a more potent inhibitory effect on asthma responses, which may be related to the increase in plasmacytoid DC recruitment. These results improve our understanding of the cellular basis of Der p 2 BCG-mediated inhibition of asthma.

Copyright $\odot 2012$ S. Karger AG, Basel

\section{Introduction}

Asthma is a chronic airway inflammatory disorder which is associated with enhanced T helper (Th) type 2 responses. It is characterized by airway inflammation, eosinophil infiltration, mucin hypersecretion and airway hyperresponsiveness (AHR). The incidence of asthma has dramatically increased over the past decades in the developed world [1]. Current asthma drug treatments, e.g. glucocorticoids, $\beta_{2}$-agonists, anticholinergics, leu-

S.-Y.Q., H.-F.Q.-Y. and Y.-L.H. contributed equally to this study. 
kotriene modifiers and adoptive transfer of stem cells, are effective in controlling and relieving asthma symptoms. However, these drugs or methods do not affect the intrinsic immune system $[2,3]$. A vaccine which can affect the underlying immune response and suppress allergic responses may be an alternative therapy for asthma.

Several studies have demonstrated that mycobacterial infection could inhibit de novo and established airway allergic responses in a murine model. These were characterized by reduced allergen-driven Th2-like cytokine production and eosinophil inflammation of the airways $[4,5]$. Previously, a recombinant bacille Calmette-Guérin (rBCG) was constructed in our laboratory which expressed Der $p$ 2 of house dust mites. It was confirmed that Der $\mathrm{p} 2$ rBCG effectively suppressed asthmatic airway inflammation [6]. There are two possible mechanisms: (1) Der p 2 rBCG elicits an immune deviation from Th2 to Th1, and (2) Der $\mathrm{p} 2$ rBCG induces antigen-specific regulatory $\mathrm{T}$ cells $\left(\mathrm{T}_{\text {regs }}\right)$. The T-cell immune response pattern could be regulated by Der $\mathrm{p} 2$ rBCG immunization in allergic inflammation. However, the exact cellular mechanism remains unclear.

DCs are the major target cells of mycobacterial infection [7]. It was reported that DCs played an important role in BCG infection-mediated inhibition of allergy to ragweed [8]. In addition, intranasal administration of BCG modulated ovalbumin (OVA)-pulsed bone marrowderived stem cells, increased the number of Foxp3+ cells in bronchial lymph nodes and reduced OVA-induced eosinophilic airway inflammation [9]. Hence, we hypothesized that DCs might also play a central role in Der $\mathrm{p} 2$ rBCG-mediated inhibition of allergic airway inflammation. In this study, we compared the immune-regulatory role of Der $\mathrm{p} 2 \mathrm{rBCG}$-DCs in a murine model of asthma and investigated possible mechanisms.

\section{Materials and Methods}

\section{Animals}

Healthy female C57BL/6 mice (6-8 weeks of age) were purchased from the Experimental Animal Center of the Fourth Military Medical University (Xi'an, China). All mice were bred and maintained under specific pathogen-free conditions. All study protocols were approved by the Animal Experiment Administration Committee of the Fourth Military Medical University.

\section{Der $p 2$ rBCG and Immunization Strategy}

Der $\mathrm{p} 2 \mathrm{rBCG}$ was constructed by Prof. Shi as described previously [10]. BCG was grown in Middlebrook 7H9 medium (Difco Laboratories, Detroit, Mich., USA) supplemented with $0.5 \%$ glycerol, $0.05 \%$ Tween- 80 and $10 \%$ albumin-dextrose-catalase at $37^{\circ} \mathrm{C}$. It was harvested at $\log$ growth phase and frozen at $-70^{\circ} \mathrm{C}$.
On day 0 , mice were intraperitoneally injected with $1 \times 10^{6}$ colony-forming units of Der p 2 rBCG or BCG. On day 42, mice were sacrificed for DC sampling. DCs were used for either adoptive transfer or FACS analysis.

DCs were prepared by CD11c magnetic beads. Briefly, the abdominal draining lymph nodes (DLNs) were minced and filtered through a nylon filter. DCs were separated by CD11c magnetic beads (Miltenyi Biotec, Bergisch Gladbach, Germany) from DLN cell suspensions. Another group treated with physiological saline (PS) served as control. The purity of the CD11c+ cells used in the experiment was $>90 \%$.

\section{Asthma Model and Study Design}

Before DC transfer, mice were randomly divided into six groups: the control group, positive control group, Der p 2 rBCGDC group, BCG-DC group, PS-DC group and Der p 2 rBCGDC+CD25 antibody group. Each group had 6 mice.

In the control group, mice were treated, sensitized and challenged with PS, which served as negative control. In the positive control group, mice were sensitized with Der $\mathrm{p} 2$ absorbed to potassium alum (i.p.) and challenged with Der p 2 (i.n.) without any DC transfer. They served as positive control.

In the other groups, mice were first sensitized with $100 \mu \mathrm{g}$ Der p 2 (Indoor Biotechnologies, Charlottesville, Va., USA) absorbed to $9 \%$ potassium alum (A1577; Sigma-Aldrich) intraperitoneally on days 28 and 35. Abdomen DLN DCs from mice immunized with Der p 2-rBCG (Der p 2 rBCG-DCs), BCG (BCG-DCs) or PS (PS-DCs) were irradiated (10 Gy) and injected intranasally $(1 \times$ $10^{6} \mathrm{cell} /$ mouse) into the sensitized mice on day 42 . Anti-CD25 monoclonal antibodies (PC61, ATCC) were injected intraperitoneally on days 43 and 45 in the Der p 2 rBCG-DC+PC61 group. The mice were challenged with $100 \mu \mathrm{g}$ Der $\mathrm{p} 2$ i.n. on days 46, 47 and 48 , and were sacrificed $48 \mathrm{~h}$ after the last challenge.

\section{Bronchoalveolar Lavage Fluid}

Bronchoalveolar lavage fluid (BALF) was collected as previously described [6]. In brief, $0.3 \mathrm{ml}$ PBS were injected slowly into the trachea three times, and BALF was centrifuged at $1,200 \mathrm{~g}$ for $5 \mathrm{~min}$; the pellets were resuspensed in PBS for cellular analysis. The supernatants were stored at $4{ }^{\circ} \mathrm{C}$ for ELISA. The concentrations of IFN- $\gamma$, IL-4, IL-5, IL-10, IL-13, TGF- $\beta$ and eotaxin in BALF were assayed by ELISA kits (Neobioscience, ShenZhen, China). The total number of cells in BALF was counted by a hemocytometer. Lymphocytes and eosinophils were counted based on their morphology and staining characteristics.

\section{Lung Histology}

For histopathology, lungs were fixed with $10 \%$ buffered formalin after BALF collection. Lung samples were embedded in paraffin and then sectioned $(4 \mu \mathrm{m})$. Sections were stained with hematoxylin and eosin (H\&E) for analyzing airway inflammation and pathological changes. Goblet cells were stained with Alcian blue-periodic acid schiff (AB-PAS). The area of bronchi and the PAS-stained areas were measured, and the histological mucus index was quantified by a previously described formula (PAS stained area/bronchial circumference area) [8].

\section{Determination of $A H R$}

Briefly, mice were anesthetized and then intubated and mechanically ventilated (120 breaths/min, $0.2 \mathrm{ml}$ tidal volume). Sub- 
sequently, mice were paralyzed with decamethonium and $50 \mathrm{mg} /$ kg i.p. acetylcholine (Ach) were injected. Airway responsiveness is expressed as the airway pressure-time index [11].

\section{Mixed Lymphocytes Reaction}

Purified naive CD4+ T cells were isolated from abdominal DLNs in normal mice by magnetic beads [12]. The purity of the CD $4+$ cells was $>90 \%$. Naive T cells $\left(5 \times 10^{5}\right.$ cells $\left./ \mathrm{ml}\right)$ were cocultured with DCs which were previously treated with Der $\mathrm{p} 2$ rBCG or BCG or PS for $72 \mathrm{~h}$. Der $\mathrm{p} 2$ antigen was added at the beginning of the DC-T-cell co-culture. After $72 \mathrm{~h}$, the supernatants were collected for ELISA cytokine assays, and T cells were collected for FACS analysis.

\section{FACS Analysis}

Mice were sacrificed $48 \mathrm{~h}$ after the last challenge. Thoracic lymph nodes (TLNs) were collected and minced. Cell suspensions were filtered through a nylon membrane and resuspended in FACS buffer (PBS, $2 \%$ FCS, $0.05 \% \mathrm{NaN}_{3}$ ). In mixed lymphocyte reaction assays, whole cells were collected and resuspended in FACS buffer. For surface staining, cells were labeled with PE-antimouse CD4 antibody (GK1.5; BD PharMingen, San Diego, Calif., USA) and FITC-anti-mouse CD25 antibody (7D4; BD Pharmingen). For Foxp3 staining, a kit (Bioscience) containing the monoclonal antibody FKJ-16s was used according to the manufacturer's instructions. The cells were analyzed with a FACSCalibur ${ }^{\mathrm{TM}}$ flow cytometer (BD Immunocytometry Systems, San Jose, Calif., USA). Dead cells were excluded by staining with propidium iodide and gating. The data were analyzed using CellQuest software.

To observe the effects of Der p 2 rBCG on phenotype differentiation on DCs, abdomen DLN single-cell suspensions were prepared. Cells were labeled with the following antibodies, CD-80 (16-110A1), CD-86 (GL-1), MHC II (KH174), FITC-CD11c (N418), APC-B220 (RA3-6B2; BD Pharmingen) and PE-Siglec-H antibody (551; Biolegend, San Diego, Calif., USA).

\section{Real-Time PCR}

Firstly, TLN cell suspensions were collected and minced as described. Purified $\mathrm{T}_{\text {regs }}$ sere isolated from TLN cell suspensions using the CD4+CD25+ regulatory $\mathrm{T}$ cell isolation kit (MACS; Miltenyi Biotec). In in vitro studies, we isolated $\mathrm{T}_{\text {reg }} \mathrm{s}$ in the mixed lymphocyte reaction systems. Then total RNA was extracted from $\mathrm{T}_{\text {reg }} \mathrm{s}$ using TRIzol reagent (Invitrogen, Carlsbad, Calif., USA) according to the protocols and then reverse transcribed with a kit (Toyobo, Osaka, Japan). Real-time PCR was performed using SYBR Premix Ex Taq (Takara, Otsu, Japan) and the ABI PRISM 7300 real-time PCR equipment. $\beta$-Actin was used as an internal control. Sequences of primers were as follows: TGF- $\beta$ forward: GTGTGGAGCAACATGTGGAACTCTA, and reverse: TTGGTT CAGCCACTGCCGTA, and $\beta$-actin forward: ATCCGTAAAGACCTCTATGCCAAC, and reverse: TGGAGCCACCGATCCACA.

\section{Statistical Analysis}

Data were expressed as means \pm SD. Statistical analyses were performed by single-factor ANOVA for multiple groups and Student's unpaired t test for two groups. A value of $\mathrm{p}<0.05$ was assumed to be significantly different.

\section{Results}

Adoptive Transfer of Derp 2 rBCG-DCs Suppresses Airway Inflammation and $A H R$ in vivo

Mice transferred the Der $\mathrm{p} 2$ rBCG-DCs or BCG-DCs exhibited significantly less airway cell infiltration than the PS-DCs group, especially less eosinophils. However, in the Der $\mathrm{p} 2 \mathrm{rBCG}-\mathrm{DC}+\mathrm{PC} 61$ group, the cell number in BALF was much higher than in Der p 2 rBCG-DC, but similar to the positive control group (fig. 1a). Moreover, there was no significant difference in neutrophil numbers between the Der $\mathrm{p} 2 \mathrm{rBCG}$ and BCG groups (data not shown). Therefore, the suppressive effect on local airway inflammation was higher in Der p 2 rBCG-DCs than BCG-DCs, and this effect was inversed by PC61.

$\mathrm{H} \& \mathrm{E}$ staining was used to characterize cellular infiltration, and AB-PAS staining was used to evaluate bronchial mucus production in the tracheal cavity. Der $\mathrm{p} 2$ rBCG-DCs and BCG-DCs recipients displayed significantly reduced peribronchial and perivascular cellular infiltration, and there was less mucus staining in lung tissue than in the PS-DCs group. However, PC61 treatment markedly reversed lung tissue inflammation (fig. 1b). The histological mucus index (which indicates bronchial mucus production) was significantly lower in Der p 2 BCGDC and BCG-DC recipients than in PS-DC recipients. Furthermore, Der p 2 rBCG-DCs had more inhibitory effects on mucus production than BCG-DCs, and this effect was associated with $\mathrm{T}_{\text {reg }}$ induction (fig. 1c).

We examined the capacity of Der $\mathrm{p} 2 \mathrm{rBCG}-\mathrm{DC}$ to regulate AHR, which was assessed by the airway pressure-time index. In the positive control mice, ACh response was increased compared with the control group. Der $\mathrm{p} 2 \mathrm{rBCG}-\mathrm{DC}$ and BCG-DCs significantly inhibited $\mathrm{ACh}$ responsiveness. Interestingly, transfer of Der $\mathrm{p} 2$ rBCG-DCs was more effective than that of BCG-DCs, and $\mathrm{T}_{\text {reg }}$ depletion suppressed this effect (fig. 1d).

\section{Der $p 2$ rBCG-DCs Suppressed the Production of Th2}

Cytokines and Induced Immunoregulatory Molecules

The supernatants of BALF were collected to investigate the mechanisms of immune regulation of Der $\mathrm{p} 2$ rBCG-DCs. IL-4, IL-5, IL-13, IFN- $\gamma$, IL-10, TGF- $\beta$ and eotaxin concentrations were assayed by ELISA. As shown in figure 2a, IL-4, IL-5, IL-13 and eotaxin levels were decreased and INF- $\gamma$, TGF- $\beta$ and IL-10 levels were significantly increased in the Der p 2-rBCG-DC and BCG-DC groups compared with positive control and PS-DC groups. In addition, the decreases in IL-5, IL-13 and eotaxin levels were more pronounced in Der $\mathrm{p} 2 \mathrm{rBCG}$-DCs 

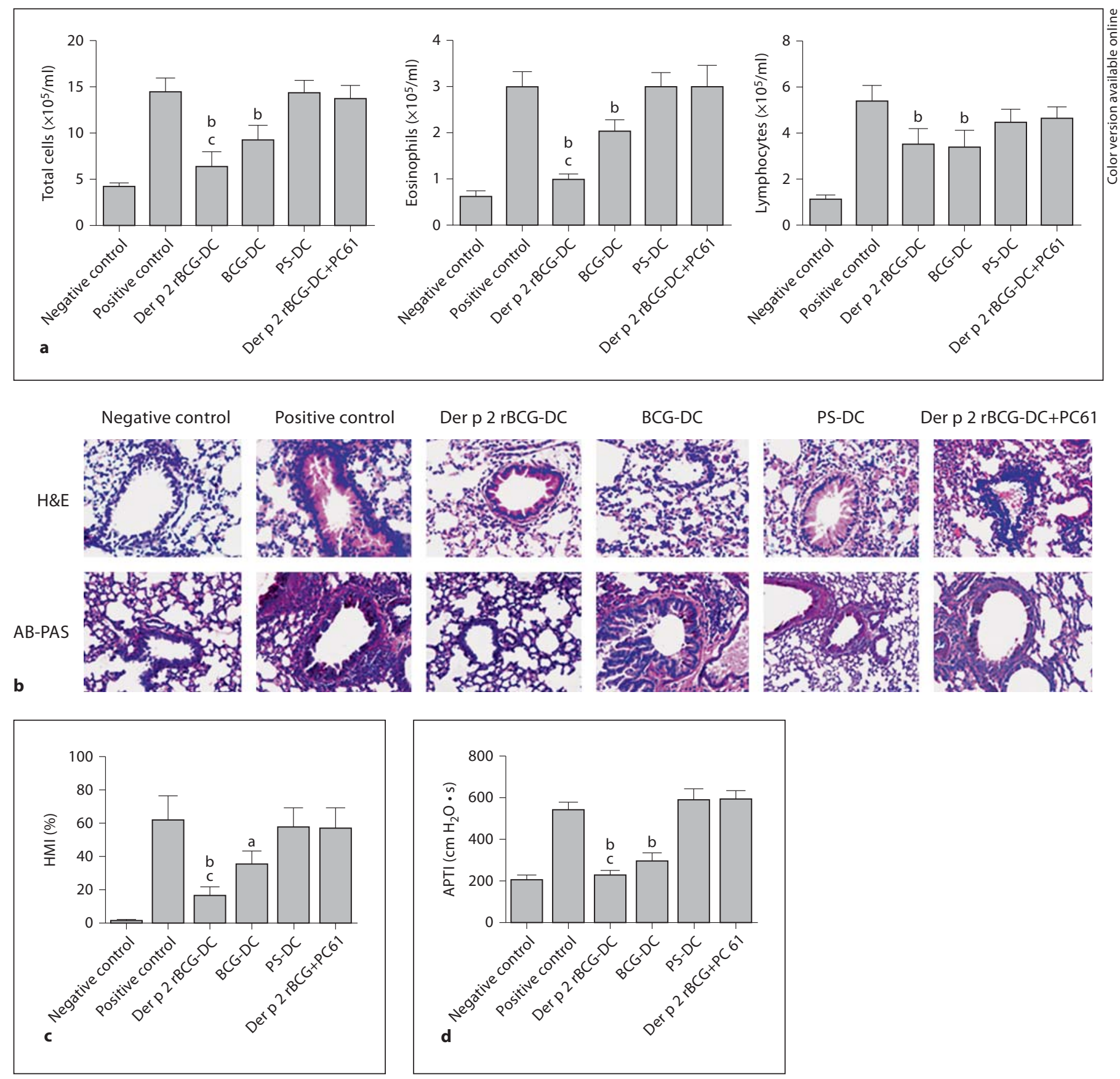

Fig. 1. Adoptive transfer of Der p 2 rBCG-DCs suppressed airway inflammation and AHR in vivo. The mice were sensitized with Der p $2(100 \mu$ g i.p.) absorbed with $9 \%$ potassium alum on days 28 and 35. DCs $\left(1 \times 10^{6}\right)$ from mice immunized with Der p 2 rBCG, BCG or PS were transferred intranasally into the sensitized mice on day 42. Anti-CD25 monoclonal antibodies (PC61; ATCC) were injected intraperitoneally on days 43 and 45 . The mice were challenged with Der p 2 (100 $\mu \mathrm{g}$ i.n.) on days 48, 49 and 50. The experiments were performed $48 \mathrm{~h}$ after the last challenge. a Pulmonary cellular infiltration was examined by BALF cell counts.
The absolute numbers of total cells and of each cell type are shown. b Lungs were fixed in $10 \%$ buffered formalin, and lung sections were examined by H\&E or AB-PAS staining. $\mathbf{c}$ The histological mucus index (HMI) of each group is shown. d AHR to methacholine. ${ }^{\mathrm{a}} \mathrm{p}<0.05,{ }^{\mathrm{b}} \mathrm{p}<0.01$, vs. PS-DC group, ${ }^{\mathrm{c}} \mathrm{p}<0.05$ vs. BCG-DC group. In all figures, the asthma model group was replaced by the positive control group and the control group was replaced by the negative control group. APTI = Airway pressuretime index. 


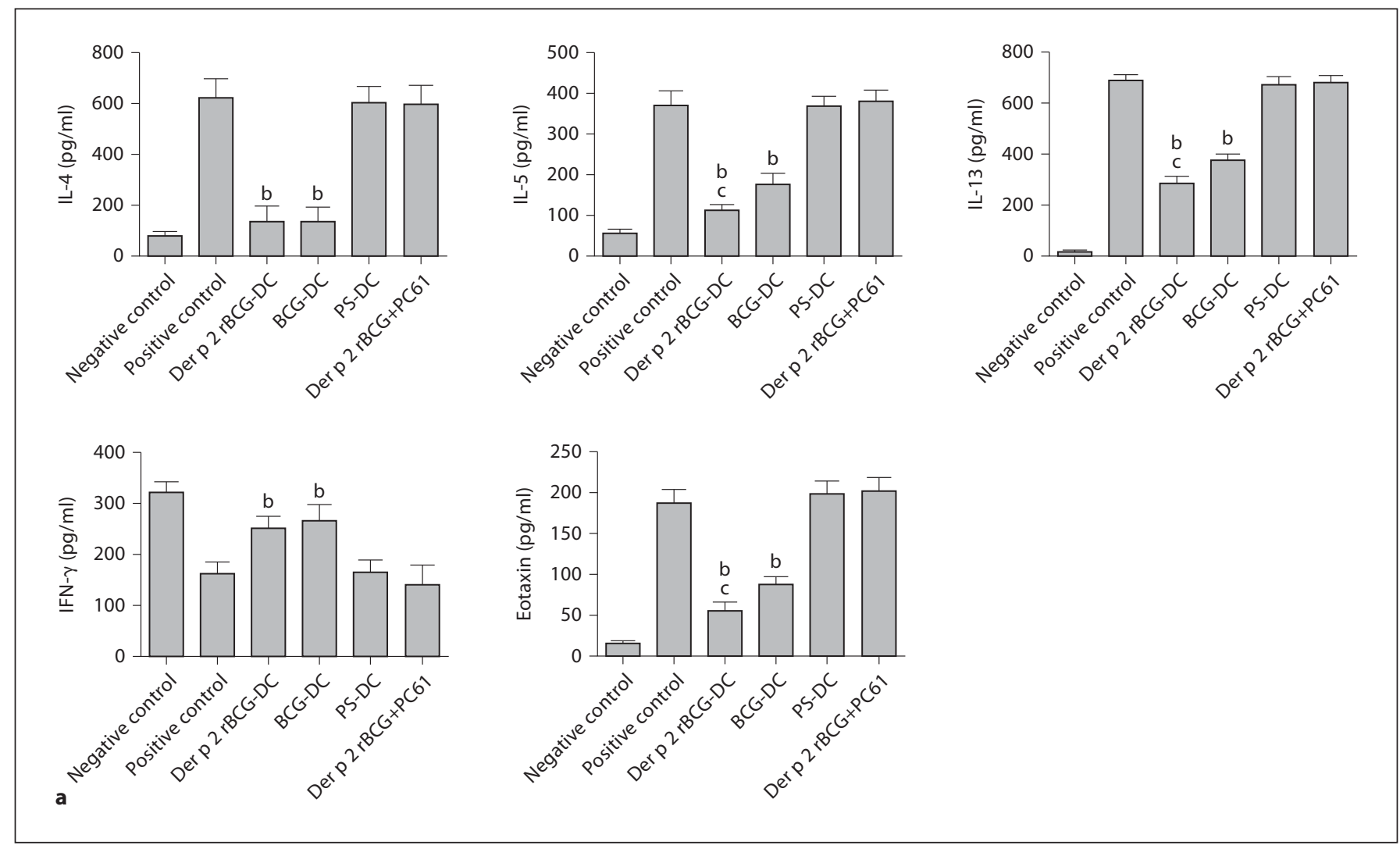

Fig. 2. Der p 2 rBCG-DCs suppressed the production of Th2 cytokines and induced immunoregulatory molecules. BALF were collected $48 \mathrm{~h}$ after the last challenge. Th1/ Th2 levels and immunoregulatory cytokines in BALF (pg/ml) were assayed by ELISA, and IL-4, IL-5, IL-13 and eotaxin (a) and IL-10 and TGF- $\beta$ (b) in BALF are determined. ${ }^{b} \mathrm{p}<0.01$, vs. PS-DC group, ${ }^{\mathrm{c}} \mathrm{p}<0.05$ vs. BCG-DC group.

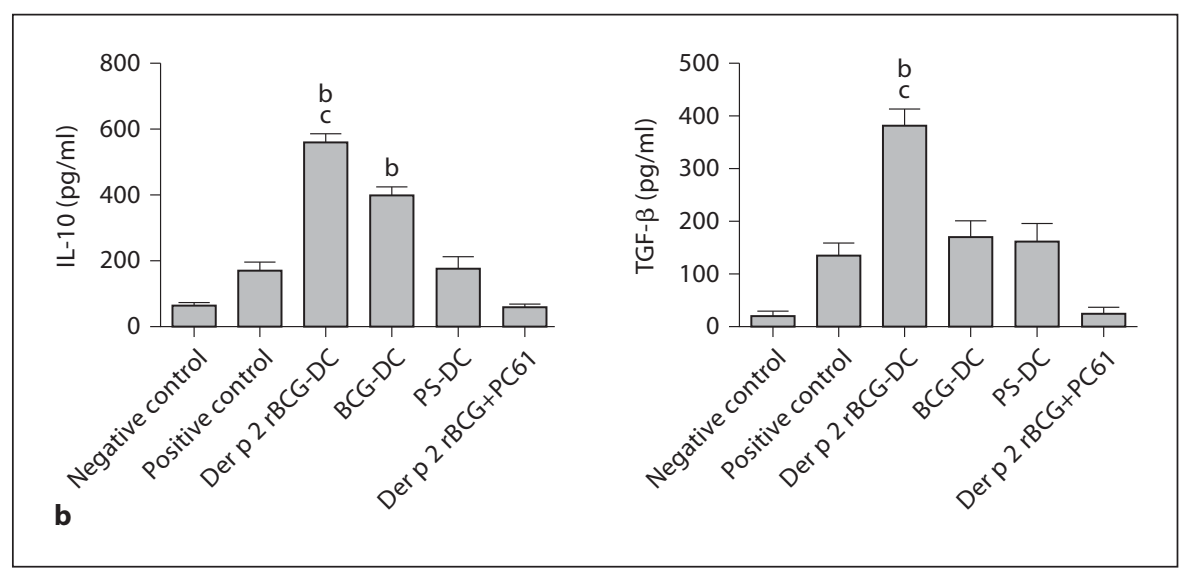

than in BCG-DCs. TGF- $\beta$ and IL-10 levels were also significantly higher in the Der $\mathrm{p} 2$ rBCG-DC group compared with the BCG-DC group (fig. 2b). Der p 2 rBCGDCs showed a more potent immunoregulatory effect than BCG-DCs.

We previously described that Der p 2 rBCG induced a subtype of $\mathrm{T}_{\text {reg }} \mathrm{s}$ [6]. To understand the mechanism of $\mathrm{T}_{\text {reg }}$ functions in this process, PC61 was used to deplete $\mathrm{CD} 25+$ cells in vivo. The results showed that the percent- age of CD4+CD25+Foxp3+ in Der p 2 rBCG-DCs+PC61 mice was greatly decreased. The symptoms of asthma were more severe. This included inflammatory cell infiltration of the airways, Th2 cytokine production, goblet cell hyperplasia and mucus production; PC61 increased IL-4 and IL-5 levels, and significantly suppressed IFN- $\gamma$, IL-10 and TGF- $\beta$ levels. 

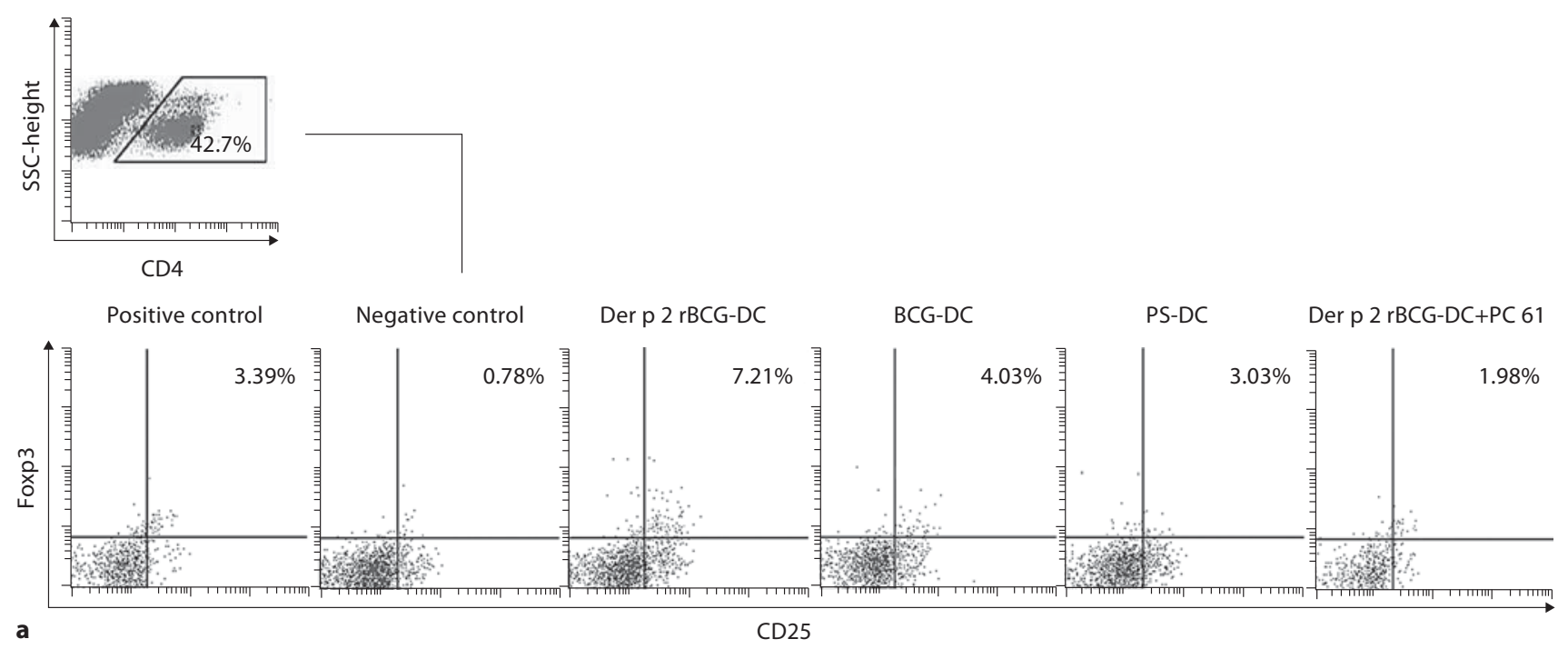

Fig. 3. Der p 2 rBCG-DCs induced CD4+CD25+Foxp3+ $\mathrm{T}_{\text {reg }} \mathrm{s}$ in vivo and in vitro. In vivo, mouse TLNs were collected and single-cell suspensions were resuspended in PBS. In vitro, naive $\mathrm{CD} 4+\mathrm{T}$ cells were co-cultured with Der p 2 rBCGDCs, BCG-DCs and PS-DCs. CD4, CD25 and Foxp3 expression was analyzed by FACS. a Mouse TLNs were collected and single-cell suspensions were resuspended in PBS. CD4, CD25 and Foxp3 expression was analyzed by FACS. Positive control group vs. control group: $\mathrm{p}<0.05$; Der $\mathrm{p} 2$ rBCG-DC group and BCG-DC and PS-DC group vs. positive control group, Der $\mathrm{p} 2$ rBCG-DC group vs. BCG-DC group: $\mathrm{p}<$ 0.05 ; vs. positive control group: $\mathrm{p}<0.05$, and vs. PS-DC group: $\mathrm{p}<0.05$. b Statistical analysis of absolute $\mathrm{T}_{\text {reg }}$ numbers in vivo. c Naive CD4+ T cells were co-cultured with Der p 2 rBCG-DCs, BCG-DCs and PS-DCs. CD4, CD25 and Foxp3 expression was analyzed by FACS. ${ }^{\mathrm{a}} \mathrm{p}<0.05$, ${ }^{\mathrm{b}} \mathrm{p}<0.01$ vs. PS-DC group, ${ }^{\mathrm{c}} \mathrm{p}<0.05$ vs. BCG-DC group.
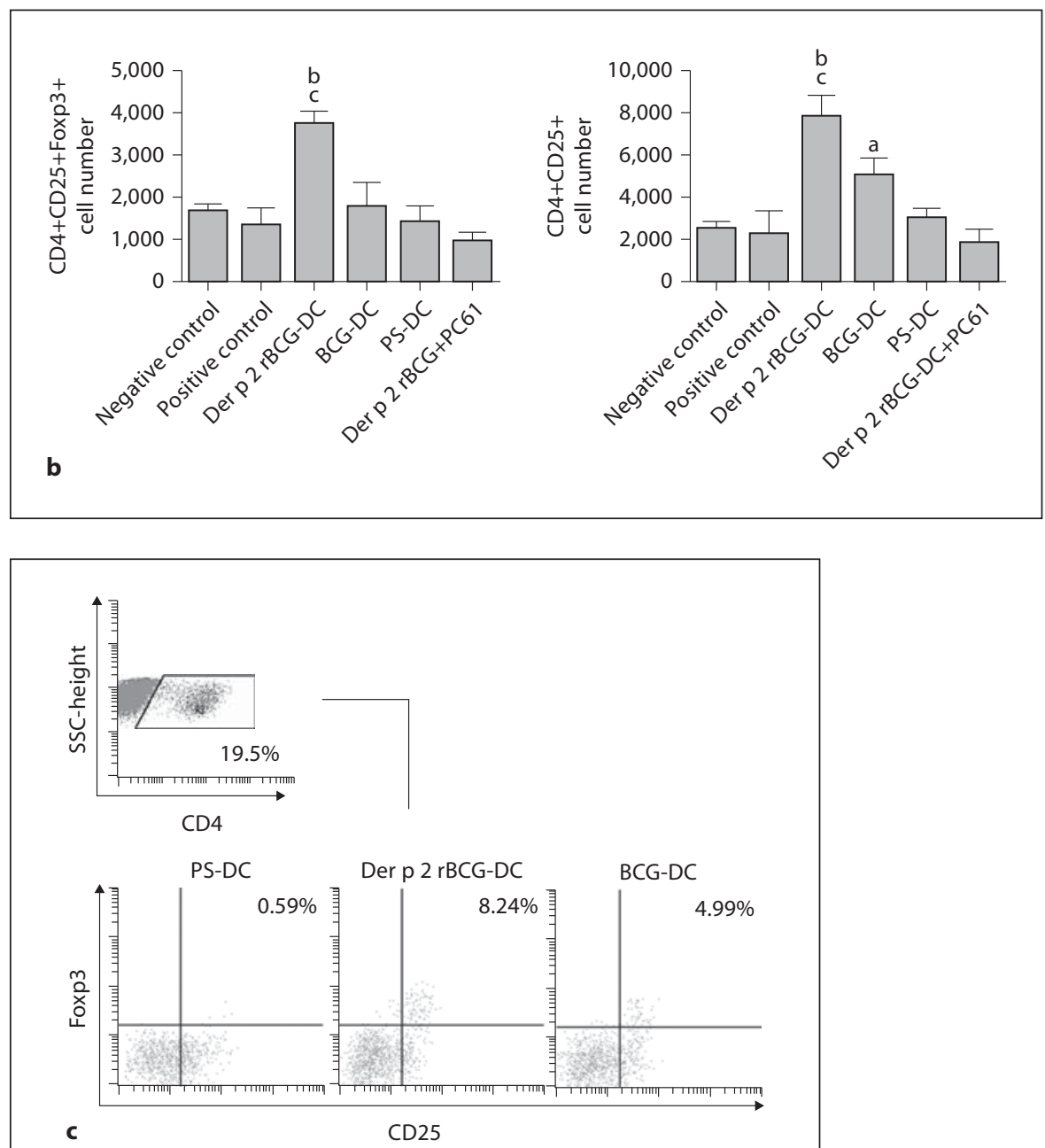

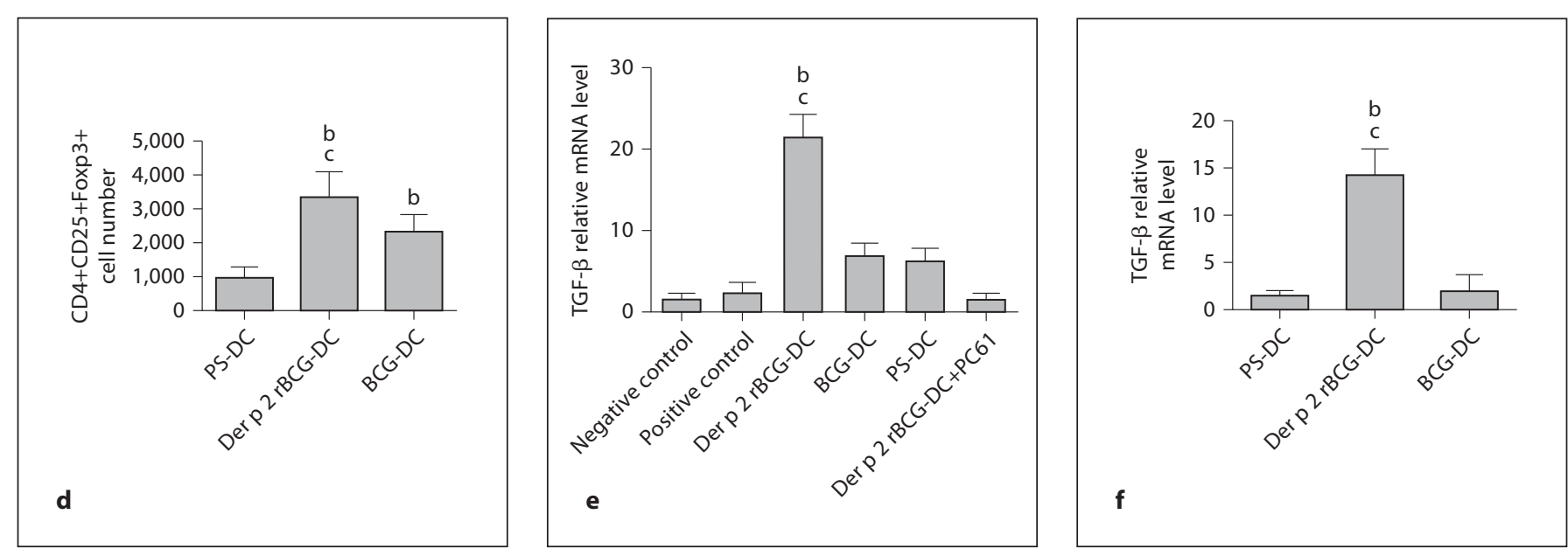

Fig. 3. Der p 2 rBCG-DCs induced CD $4+C D 25+F o x p 3+T_{\text {reg }} \mathrm{s}$ in vivo and in vitro. In vivo, mouse TLNs were collected and singlecell suspensions were resuspended in PBS. In vitro, naive CD4+ T cells were co-cultured with Der p 2 rBCG-DCs, BCG-DCs and PS-DCs. CD4, CD25 and Foxp3 expression was analyzed by

Der p 2 rBCG-DCs Induced CD4+CD25+Foxp3+ $T_{\text {reg }} s$ in vivo and in vitro

The mice were sensitized with Der $\mathrm{p} 2$ and then adoptively transferred with irradiated Der p 2 rBCG-DCs, BCG-DCs or PS-DCs, respectively. Subsequently, they were challenged with Der $\mathrm{p} 2$. The ratio of CD4+CD25+Foxp3+ $\mathrm{T}_{\text {reg }}$ in total TLN CD4+ cells was calculated. In Der p 2 rBCG-DCs and BCG-DCs, the $\mathrm{CD} 4+\mathrm{CD} 25+\mathrm{Foxp} 3+\mathrm{T}_{\text {reg }}$ ratio was significantly increased $(\mathrm{p}<0.001)$, and Der $\mathrm{p} 2$ rBCG-DCs induced more $\mathrm{CD} 4+\mathrm{CD} 25+$ Foxp3 $+\mathrm{T}_{\text {regs }}(\mathrm{p}<0.05)$. In the Der $\mathrm{p} 2$ rBCG-DC+PC61 group, the number was much lower than in the Der $\mathrm{p} 2$ rBCG-DC group but similar to the positive control group (fig. 3a, b).

In in vitro studies, naive CD4+ T cells were isolated from DLNs in normal mice, and then co-cultured with DCs which were previously treated with Der $\mathrm{p} 2 \mathrm{rBCG}$ or BCG or PS for $72 \mathrm{~h}$. Der $\mathrm{p} 2$ antigen was added at the beginning of the DC-T-cell co-culture. FACS revealed that Der p 2 rBCG-treated DCs induced more CD4+CD25+Foxp3+ T cells than BCG-DCs and PS-DCs $72 \mathrm{~h}$ after the co-culture ( $<<0.05$; fig. $3 \mathrm{c}, \mathrm{d})$.

Real-time PCR indicated TGF- $\beta$ and IL-10 levels of $\mathrm{T}_{\text {reg }}$ s from Der $\mathrm{p} 2$ rBCG-DCs were higher in vivo compared with PS-DCs (fig. 3e), implying that $\mathrm{T}_{\text {reg }} \mathrm{s}$ are the source of TGF- $\beta$ and IL-10 in the Der p 2 rBCG-DCs. In in vitro studies, TGF- $\beta$ and IL-10 mRNA were also elevated in Der p 2 rBCG-DCs (fig. 3f).
FACS. d Statistical analysis of absolute $T_{\text {reg }}$ numbers in vitro. e, $f T_{\text {reg }}$ sere collected and the TGF- $\beta$ mRNA level was determined by real-time PCR in vivo and in vitro. ${ }^{b} \mathrm{p}<0.01$ vs. PC-DC group, ${ }^{\mathrm{c}} \mathrm{p}<0.05$ vs. BCG-DC group.

\section{Effects of Derp 2 rBCG on DC Phenotypes}

Der $\mathrm{p} 2$ rBCG-DCs expressed higher levels of the costimulatory molecules CD80, CD86 and MHC II than the control group, but there were no significant differences between BCG and Der p 2 rBCG. Thus, Der p 2 rBCG and BCG had similar effects in inducing the maturation of DCs in vivo (fig. 4a). We found that the production of cytokines, including IL-12 and IL-10, induced by BCG- and Der p 2 rBCG-stimulated DCs was the same (data not shown).

Because plasmacytoid DCs (pDC) played an important role in inhaled antigen tolerance, we speculated that pDC might be involved in the regulatory role of Der $\mathrm{p} 2$ rBCG-DCs. Mice were immunized with Der p 2 rBCG, BCG or PS on day 0 . The whole DLN cells were collected and analyzed by FACS on day 42 . The relative ratios and the absolute numbers of CD11c+B220+ Siglec-H+ pDCs per DLN were also higher in the Der $\mathrm{p} 2$ rBCG group than in the BCG and control groups (fig. $4 \mathrm{~b}, \mathrm{c}$ ). However, the number of conventional DCs (cDCs) did not differ among those three groups (fig. $4 \mathrm{~d}$ ).

\section{Discussion}

Our previous studies showed that Der $\mathrm{p} 2$ rBCG inhibited allergen-induced airway inflammation. Those protective effects were associated with both immune devia- 
Fig. 4. The effect of Der p 2 rBCG on DC phenotypes. a Surface expression of CD80, CD86 and MHC II of DCs was examined with FACS in different groups (light gray: isotype; bold line: Der p 2 rBCG; dark grey, BCG, and dotted line, control). b-d Identification of DC subsets recruited to the DLNs; 42 days after immunization with Der p 2 rBCG, BCG or PS, DLN cells were isolated, and pDCs (CD11c+ B220+) and cDCs (CD11c+B220-) were sorted and the absolute numbers of $\mathrm{cDCs}$ assessed. The absolute numbers of pDCs (CD11c+ $\mathrm{B} 220+$ Siglec-H+) in each DLN were also determined. Data are representative of three independent experiments. ${ }^{\mathrm{b}} \mathrm{p}<0.01$ vs. PS group, ${ }^{c} \mathrm{p}<0.05$ vs. BCG group.
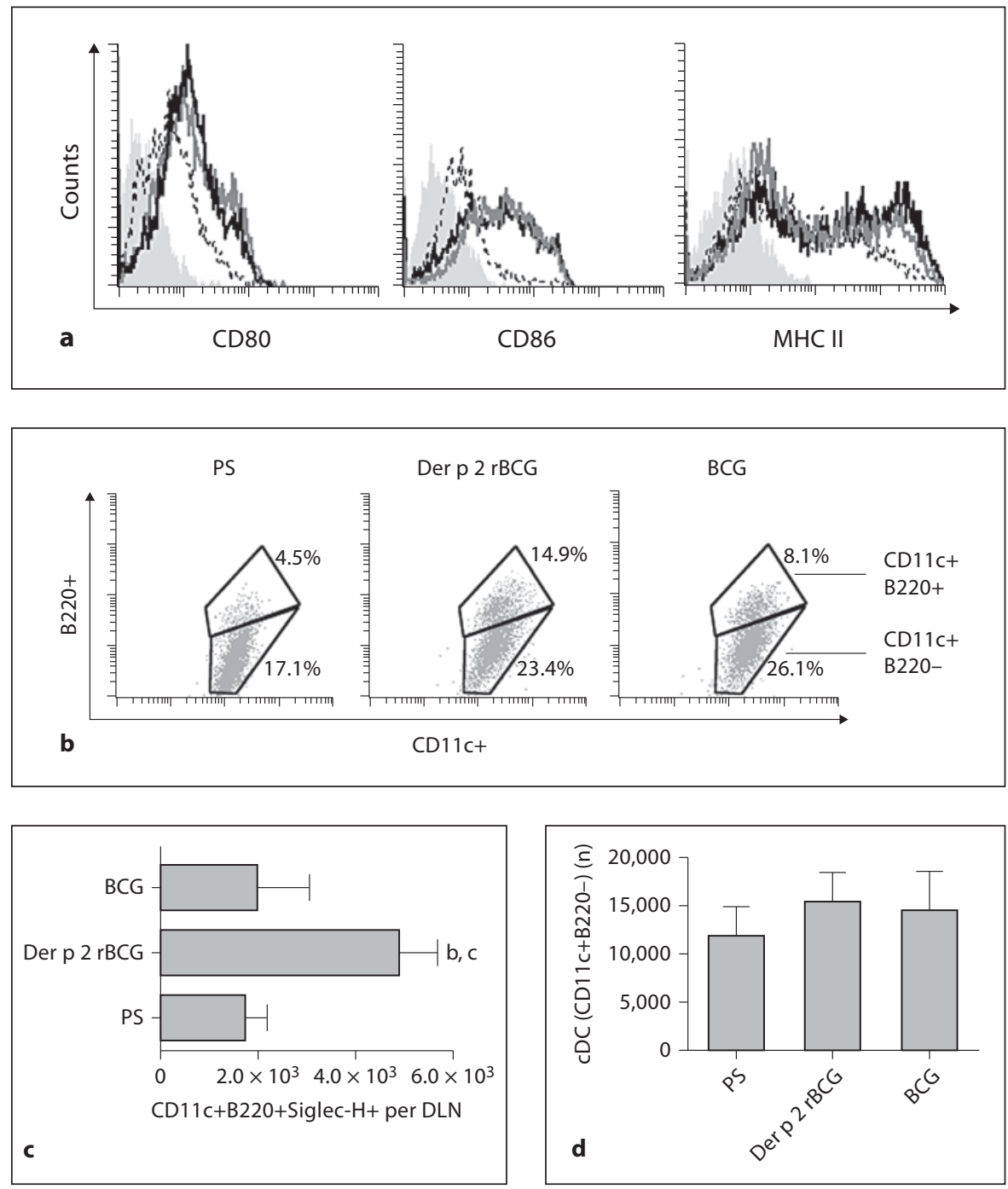

tion and immune suppression [6]. However, the exact cellular immunoregulatory mechanisms of Der $\mathrm{p} 2 \mathrm{rBCG}$ remain to be determined. In this study, DCs from Der $\mathrm{p}$ 2 rBCG-immunized mouse reduced Th2-like responses, eosinophil number, airway inflammation, mucus hypersecretion and AHR induced by allergen challenge. Der $\mathrm{p}$ 2 rBCG-DCs induced CD4+CD25+Foxp3+ $\mathrm{T}_{\text {regs }}$ s Furthermore, immunization with Der p 2 rBCG recruited more pDCs than BCG in the DLNs. All the results indicated that DCs played a vital role in the immunoregulatory effects of Der p 2 BCG.

DCs are composed of several heterogeneous subpopulations. Several DC subpopulations, such as DC1-like cells $[13,14]$, regulatory DC $[15,16]$ and pDCs $[17,18]$, can inhibit allergic inflammation through different mecha- nisms. In addition, allergen-DNA-modified DCs can also suppress the established Th2 responses [19]. We reported that Der $\mathrm{p} 2$ rBCG-infected DCs reduced allergic airway inflammation. Thus, immunotherapy based on antigenmodified DCs may be feasible and efficient for asthma treatment.

DCs are one of the major target cells of mycobacterial infection [7]. Several studies in animals revealed the immunoregulatory effect of BCG-infected DCs in allergeninduced asthma. Intranasal administration of BCGmodulated OVA-pulsed bone marrow-derived stem cells increased the number of Foxp3+ cells in bronchial lymph nodes and reduced OVA-induced eosinophilic airway inflammation [9]. A very recent report confirmed that adoptive transfer of DCs from the BCG-infected mice to 
ragweed-sensitized/challenged mice inhibited allergic airway inflammation [8]. In accordance with the above studies, we confirmed that both BCG- and Der p 2 rBCGinfected DCs suppressed Der p 2-induced allergic Th2 responses. The potent inhibitory effects of Der p 2 rBCG on asthmatic airway inflammation was also mediated by DCs.

Previous studies reported that Der $\mathrm{p} 2 \mathrm{rBCG}$ induced a CD4+CD25+Foxp3+ T-cell subtype, which suppressed proliferation of Th2 effector cells in vitro in an antigenspecific manner [6]. In this study, Der p 2 rBCG-DCs also induced the CD4+CD25+Foxp3+ T-cell subtype in vivo and in vitro. In order to understand $\mathrm{T}_{\text {reg }}$ functions in this process, $\mathrm{PC} 61$ was used to deplete CD25+ cells in vivo. The results showed that the percentage of CD4+CD25+Foxp3+ in Der p 2 rBCG-DCs+PC61 mice was markedly decreased. The symptoms of asthma were greatly aggravated. This included inflammatory cell infiltration of the airways, Th2 cytokine production, goblet cell hyperplasia and mucus production. PC61 increased IL- 4 and IL- 5 levels, and IFN- $\gamma$, IL-10 and TGF- $\beta$ levels were significantly decreased.

Der p 2 rBCG might modify DC functions and then induce an antigen-specific $\mathrm{T}_{\text {reg }}$ subtype. Der $\mathrm{p} 2$ rBCGDCs induced more CD4+CD25+Foxp3+ $\mathrm{T}_{\text {reg }}$ s than BCGDCs. Real-time PCR results showed that $\mathrm{T}_{\text {reg }} \mathrm{s}$ from Der $\mathrm{p}$ 2 rBCG-DCs had higher TGF- $\beta$ and IL-10 levels in vivo and in vitro. In addition, the inhibitory effect of Der $\mathrm{p} 2$ rBCG-DCs was inversed by PC61, which depleted $\mathrm{T}_{\text {reg }} \mathrm{s}$ in vivo. Considering our previous report, we conclude that Der $\mathrm{p} 2 \mathrm{rBCG}-\mathrm{DC}$ might induce a subtype of Der $\mathrm{p}$ 2-specific $\mathrm{T}_{\text {reg }} \mathrm{s}$, while BCG-DCs could not.

$\mathrm{T}_{\text {reg }} \mathrm{s}$ produced TGF- $\beta$ and IL-10. After PC61-induced $\mathrm{T}_{\text {reg }}$ depletion in vivo, the symptoms of asthma were more severe when challenged with Der p 2. PC61 reversed the immunomodulation of the Der $\mathrm{p} 2$ rBCG-DCs. Our results showed that PC61 significantly suppressed IL-10 and TGF- $\beta$ levels. This is in accordance with a previous report, in which CD25+ T-cell depletion decreased IL-10 production [20]. However, it has been reported that Th1 responses are more susceptibility to $\mathrm{T}_{\text {reg }}$ regulation than Th2 responses [21]. In our study, depletion of $\mathrm{T}_{\text {reg }} \mathrm{s}$ enhanced IL-4 and IL-5 levels and suppressed IFN- $\gamma$. All these results indicated that $T_{\text {reg }}$ s play a key role in the pathogenesis of asthma. All those data suggested that without $\mathrm{T}_{\text {reg }} \mathrm{s}$, Der p 2 rBCG could not regulate the immune disequilibrium through TGF- $\beta$ and IL-10.

It is generally believed that mature DCs induce immune responses, while immature DCs induce immune tolerance. However, both Der p 2 rBCG and BCG can sig- nificantly increase the expression of co-stimulatory molecules CD80, CD86 and MHC II on DLN DCs. Thus, we focused on the effect of Der $\mathrm{p} 2 \mathrm{rBCG}$ on DC subtypes.

Der $\mathrm{p} 2 \mathrm{rBCG}$ immunization recruited more pDCs in the DLNs than BCG. The pDCs exhibited powerful immunoregulatory effects and modulated the differentiation of naive $T$ cells into $T_{\text {reg }}$ s. In accord with our results, a recent study confirmed that extended freeze-drying of BCG reduced inflammation by recruiting more pDCs in the DLN [22]. Der p 2 rBCG might increase the number of pDCs at the local allergic inflammation site and then regulate Th2 immune responses. However, the exact mechanisms of the effects of Der p 2 rBCG on the DC subtypes are still under investigation.

In summary, we demonstrated that DCs played a vital role in the immuno-regulatory effects of the Der $\mathrm{p} 2$ rBCG on asthmatic airway inflammation. Compared with BCG, Der p 2 rBCG increased more pDCs in DLNs and display a more potent inhibitory effect. These results may help to better understand the cellular basis of Der $p$ 2 rBCG-mediated inhibition of asthmatic responses, which may contribute to the development of a Der p 2 rBCG vaccine.

\section{Acknowledgment}

This work was supported by grants from the National Natural Science Foundation of China (30971305).

References

1 von Mutius E, Martinez FD, Fritzsch C, Nicolai T, Roell G, Thiemann HH: Prevalence of asthma and atopy in two areas of West and East Germany. Am J Respir Crit Care Med 1994;149:358-364.

2 Ou-Yang H-F, Han X-P, Zhao F, Ti X-Y, Wu C-G: The role of bone marrow-derived adult stem cells in a transgenic mouse model of allergic asthma. Respiration 2012;83:74-80.

13 Barnes PJ: New therapies for asthma: is there any progress? Trends Pharmacol Sci 2010;31: 335-343.

-4 Yang X, Fan Y, Wang S, Han X, Yang J, Bilenki L, et al: Mycobacterial infection inhibits established allergic inflammatory responses via alteration of cytokine production and vascular cell adhesion molecule-1 expression. Immunology 2002;105:336-343.

5 Erb KJ, Holloway JW, Sobeck A, Moll H, Le Gros G: Infection of mice with Mycobacterium bovis-Bacillus Calmette-Guérin (BCG) suppresses allergen-induced airway eosinophilia. J Exp Med 1998;187:561-569. 
6 Ou-Yang HF, Hu XB, Ti XY, Shi JR, Li SJ, Qi $\mathrm{HW}$, et al: Suppression of allergic airway inflammation in a mouse model by Der $\mathrm{p} 2$ recombined BCG. Immunology 2009; 128:e343-e352.

7 Jiao X, Lo-Man R, Guermonprez P, Fiette L, Dériaud E, Burgaud S, et al: Dendritic cells are host cells for mycobacteria in vivo that trigger innate and acquired immunity. J Immunol 2002;168:1294-1301.

8 Bilenki L, Gao X, Wang S, Yang J, Fan Y, Han X, Qiu H, Yang X.: Dendritic cells from mycobacteria-infected mice inhibits established allergic airway inflammatory responses to ragweed via IL-10- and IL-12-secreting mechanisms. J Immunol 2010;184 7288-7296.

-9 Ahrens B, Gruber C, Rha RD, Freund T, Quarcoo D, Awagyan A, et al: BCG priming of dendritic cells enhances $T$ regulatory and Th1 function and suppresses allergen-induced Th2 function in vitro and in vivo. Int Arch Allergy Immunol 2009;150:210-220.

$\rightarrow 10$ Shi JR, Li Y, Qi HW, Li BH, Fan XL: Construction and identification of the E. coliBCG shuttle vector expressing lipoprotein Der $\mathrm{p} 2$ on cell wall of mycobacterium vaccae (in Chinese). Xi Bao Yu Fen Zi Mian Yi Xue Za Zhi 2003;19:132-135.
11 Levitt RC, Mitzner W: Expression of airway hyperreactivity to acetylcholine as a simple autosomal recessive trait in mice. FASEB J 1988;2:2605-2608.

-12 Feng F, Wang YC, Hu XB, Liu XW, Ji G, Chen YR, et al: The transcription factor RBP-J-mediated signaling is essential for dendritic cells to evoke efficient anti-tumor immune responses in mice. Mol Cancer 2010;9:90102.

13 Pulendran B, Smith JL, Caspary G, Brasel K, Pettit D, Maraskovsky E, et al: Distinct dendritic cell subsets differentially regulate the class of immune response in vivo. Proc Natl Acad Sci USA 1999;96:1036-1041.

14 Maldonado-López R, De Smedt T, Pajak B, Heirman C, Thielemans K, Leo O, et al: Role of CD8alpha ${ }^{+}$and CD8alpha ${ }^{-}$dendritic cells in the induction of primary immune responses in vivo. J Leukoc Biol 1999;66:242246.

15 Fujita S, Yamashita N, Ishii Y, Sato Y, Sato K, Eizumi K, et al: Regulatory dendritic cells protect against allergic airway inflammation in a murine asthmatic model. J Allergy Clin Immunol 2008;121:95-104.

16 Schlichting CL, Schareck WD, Kofler S, Weis $\mathrm{M}$ : Involvement of dendritic cells in allograft rejection new implications of dendritic cellendothelial cell interactions. Mini Rev Med Chem 2007;7:423-428.

$\checkmark 17$ de Heer HJ, Hammad H, Soullié T, Hijdra D, Vos N, Willart MA, et al: Essential role of lung plasmacytoid dendritic cells in preventing asthmatic reactions to harmless inhaled antigen. J Exp Med 2004;200:89-98.
18 Ito T, Yang M, Wang YH, Lande R, Gregorio J, Perng OA, et al: Plasmacytoid dendritic cells prime IL-10-producing $\mathrm{T}$ regulatory cells by inducible costimulator ligand. J Exp Med 2007;204:105-115.

19 Klostermann B, Bellinghausen I, Böttcher I, Petersen A, Becker WM, Knop J, et al: Modification of the human allergic immune response by allergen-DNA-transfected dendritic cells in vitro. J Allergy Clin Immunol 2004;113:327-333.

20 Wu K, Bi Y, Sun K, Xia J, Wang Y, Wang C: Suppression of allergic inflammation by allergen-DNA-modified dendritic cells depends on the induction of Foxp3 + regulatory T cells. Scand J Immunol 2008;67:140-151.

-21 Cosmi L, Liotta F, Angeli R, et al: Th2 cells are less susceptible than Th1 cells to the suppressive activity of CD25+ regulatory thymocytes because of their responsiveness to different cytokines. Blood 2004;103:31173124.

22 Lagranderie M, Abolhassani M, Vanoirbeek JA, Lima C, Balazuc AM, Vargaftig BB, et al: Mycobacterium bovis bacillus CalmetteGuérin killed by extended freeze-drying targets plasmacytoid dendritic cells to regulate lung inflammation. J Immunol 2010;184: 1062-1070. 